\title{
Relación entre tipo y cantidad de carbohidratos dietarios con el perfil lipídico y ApoB100 en adultos
}

\author{
Myriam Ojeda-Arredondo*, Juliana Escobar, Martha Guerra, Martha Alvarado \\ Departamento de Nutrición y Bioquímica. Facultad de Ciencias. \\ Pontificia Universidad Javeriana, Bogotá, D.C., Colombia \\ *mojeda@javeriana.edu.co
}

Recibido: 04-03-2010; Aceptado: 13-05-2010

\section{Resumen}

Objetivo. Establecer si existe relación entre el tipo y la cantidad de carbohidratos dietarios consumidos habitualmente con los niveles plasmáticos de lípidos, lipoproteínas y apolipoproteína B100 (ApoB100) Materiales y métodos. La muestra estuvo conformada por la base de datos de 144 de profesores de la Universidad Javeriana que previamente hicieron parte de otra investigación y de la cual se empleo la información sobre su alimentación, niveles plasmáticos de lípidos, lipoproteínas y ApoB100. Para determinar la relación entre las variables dependientes e independientes se realizó análisis de correlación simple y múltiple, en los casos de no correlación, se aplicó la prueba $\mathrm{Chi}^{2}$ de independencia para relacionar el consumo de carbohidratos totales, azúcares y fibra total. Adicionalmente el análisis de varianza de un factor comparó los niveles de lípidos, lipoproteínas y ApoB100 con el consumo de carbohidratos totales, azúcares y fibra. Resultados. 76 hombres y 68 mujeres. La mayor parte de la población consume por encima de la recomendación diaria de carbohidratos totales, los hombres presentaron mayor consumo de carbohidratos tipo azúcares y más elevados los niveles plasmáticos de triglicéridos y lipoproteínas de muy baja densidad (VLDL), mientras que mujeres presentaron niveles más elevados en sus lipoproteínas de alta densidad (HDL). Al relacionar gramos de cada tipo de carbohidratos, con los niveles de lípidos, lipoproteínas y ApoB100 no se encontró correlación significativa para ninguno de los casos (p>0,05). Conclusión. No se encontró relación significativa entre el tipo y la cantidad de carbohidratos consumidos con los niveles plasmáticos de lípidos, lipoproteínas y ApoB 100 .

Palabras clave: apolipoproteína B100, carbohidratos, lípidos plasmáticos, lipoproteínas.

\begin{abstract}
Relationship between the type and quantity of dietary carbohydrates, and the lipid profile and ApoB100 in adults. Objective. To establish if there is any relationship between the type and amount of dietary carbohydrates commonly consumed and the plasmatic levels of lipids, lipoproteins and apolipoprotein B100 (ApoB100). Materials and methods. The final sample was conformed by the database of 144 lecturers of the Pontificia Universidad Javeriana who had previously participated in another survey and from which we used information on their food habits, plasmatic lipid levels, lipoproteins and ApoB100. To determine the relationship between the dependent and independent variables, simple and multiple correlation analyses were done. In the cases of no correlation we applied the independence $\mathrm{Chi}^{2}$ test to find the relationship with the consumption of total carbohydrates, sugars and total fiber. Additionally, with a one-factor analysis of variance we compared plasmatic levels of lipids, lipoproteins and ApoB100 with the consumption of total carbohydrates, sugars and fiber. Results. Most of the population consumes above the daily total carbohydrate recommendation, men presented greater carbohydrate consumption of the type of sugars and more elevated plasmatic levels of triglycerides and lipoproteins of
\end{abstract}


very low density (VLDL), whereas women presented higher levels of their high density lipoproteins (HDL). When relating grams of each type of carbohydrates with the levels of lipids, lipoproteins and ApoB100, no significant correlation was found for any of the cases ( $p>0,05)$. Conclusion. There was no significant relationship between the type and amount of carbohydrates consumed with the plasmatic levels of lipids, lipoproteins and Apo B100.

Key words: apolipoprotein B100, carbohydrates, plasmatic lipids, lipoproteins.

\begin{abstract}
Resumo
Relação entre o tipo e a quantidade de carboidratos na dieta e o perfil lipídico e ApoB100 em adultos. Objetivo. Estabelecer a possível relação entre o tipo e a quantidade de carboidratos na dieta normalmente consumida com os níveis plasmáticos de lipídios, lipoproteínas e apolipoproteína B100 (ApoB100). Materiais e métodos. A amostra consistiu de um banco de dados de 144 professores da "Universidad Javeriana" que anteriormente fizeram parte de outra pesquisa, e da qual foi utilizada a informação sobre sua alimentação, os níveis plasmáticos de lipídios, lipoproteínas e ApoB100. Para determinar a relação entre as variáveis dependentes e independentes foi realizada uma análise de correlação simples e múltipla, em casos de ausência de correlação foi usado o teste do $\mathrm{Chi}^{2}$ de independência para relacionar o consumo dos carboidratos totais, açúcares e fibra total. Além disso, a análise de variância de um fator comparou os níveis de lipídios, lipoproteínas e ApoB100 com o consumo de carboidratos totais, açúcares e fibras. Resultados. 76 homens e 68 mulheres. A maior parte da população consome acima da dose diária recomendada de carboidratos totais, os homens apresentaram maior consumo de carboidratos tipo açúcares, e mais elevados os níveis plasmáticos de triglicérides e lipoproteínas de muito baixa densidade (VLDL), enquanto que as mulheres apresentaram níveis mais elevados em lipoproteína de alta densidade (HDL). Ao relacionar gramas de cada tipo de carboidratos com os níveis de lipídios, lipoproteínas e ApoB100, nenhuma correlação significativa foi encontrada para qualquer um dos casos (p>0,05). Conclusão. Nenhuma relação significativa foi encontrada entre o tipo e a quantidade de carboidratos consumida com os níveis plasmáticos de lipídios, lipoproteínas e ApoB100.
\end{abstract}

Palavras-chave: apolipoproteína B100, carboidratos, lipídeos plasmáticos, lipoproteínas.

\section{Introducción}

Estudios epidemiológicos y de laboratorio sobre la hipercolesterolemia e hipertrigliceridemia, indican que los niveles elevados de lipoproteínas de baja densidad (LDL), son la principal causa de enfermedad cardiovascular. La apolipoproteína B, es clínicamente importante, debido a que hace parte de lipoproteínas que son consideradas aterogénicas y de promotores que desarrollan aterosclerosis. Existen dos formas la apolipoproteína B100 (ApoB100) y B48 (ApoB48). La ApoB100 está presente en las lipoproteínas de muy baja densidad (VLDL), de densidad intermedia (IDL) y de baja densidad (LDL), sin embargo, aunque existen investigaciones a nivel mundial en nuestro país no existe suficientes investigaciones que relacionen el consumo de nutrientes específicos con el comportamiento plasmático de ésta apoproteína (1).

Diversas investigaciones reportan que algunos tipos de azúcares simples, como la fructosa están relacionados con la alteración en procesos bioquímicos asociados con enfermedad del corazón debido al incremento de los niveles plasmáticos de triglicéridos, sin embargo, otros estudios indican que el efecto de la fructosa en el metabolismo de carbohidratos o lípidos, no es diferente a los de otros tipos de carbohidratos, tales como la glucosa, sacarosa o almidón (2).

En razón a lo anterior, el estudio busco establecer la relación entre el tipo y la cantidad de carbohidratos consumidos con los niveles séricos de lípidos, lipoproteínas y Apolipoproteína B100, en población adulta aparentemente sana que participó en una investigación previa titulada "Influencia de la dieta sobre los niveles plasmáticos de lípidos en sujetos adultos sanos con diferentes genotipos del gen de la FABP2" (3).

Este estudio es el inicio de nuevas investigaciones similares en población de acuerdo a grupos etáreos o población con patologías de base como por ejemplo Diabetes, Dislipidemia, Hipertensión arterial, Obesidad, entre otras.

\section{Materiales y métodos}

\section{Muestra}

La muestra estuvo conformada por 150 individuos (profesores universitarios) que participaron en la investi- 
gación anteriormente mencionada (estudio en el cual se obtuvo la muestra en forma aleatoria y con el fin de que el tamaño fuera proporcional al tamaño de cada una de las facultades de la universidad, fue un muestreo por cuotas), de los cuales se excluyeron 6 personas por no cumplir con los criterios de inclusión que consistían en ser adultos entre 20 a 65 años de edad, no tener ninguna enfermedad endocrina que pudiera estar afectando los niveles de lípidos y lipoproteínas, tales como Diabetes Mellitus e Hipo o Hipertiroidismo entre otras. Además, de no estar tomando medicamentos o estar en tratamiento dietarios. Para una muestra final de 144 individuos.

\section{Recolección de la información}

Para la realización de esta investigación, se empleó la base de datos obtenida en la investigación "Influencia de la dieta sobre los niveles plasmáticos de lípidos en sujetos adultos sanos con diferentes genotipos del gen de la FABP2" (3) en la que se trabajo con una población de profesores Javerianos aparentemente sanos, la cual contaba con información sobre aspectos de su salud, nutrición y alimentación y niveles plasmáticos de lípidos y lipoproteínas.

La frecuencia de consumo de alimentos y la evaluación cuantitativa, se obtuvo mediante la encuesta realizada en este estudio. El análisis cuantitativo de la frecuencia de consumo, se realizó mediante la Tabla de Composición de Alimentos de Medellín (4). En la evaluación e interpretación del consumo se tomo en cuenta los requerimientos estimados de energía (EER) y a partir de esto se realizó la distribución porcentual recomendada de nutrientes para la población adulta sana dados por la Nacional Academy of Sciences: Dietary References Intakes (DRIs), (5).

De la misma mannera, a partir del análisis cuantitativo de la frecuencia de consumo se relacionó la influencia del consumo de cada tipo de carbohidratos sobre los niveles plasmáticos de lípidos, lipoproteínas y ApoB 100.

Los datos de laboratorio (niveles de lípidos y lipoproteínas) fueron reportados en la investigación mencionada por el Laboratorio de Bioquímica Clínica Especializada de la Facultad de Ciencias de la Pontificia Universidad Javeriana. Los niveles de ApoB100 a pesar de no estar reportados en el estudio mencionado, fueron determinados a la misma población estudio por la Técnica de
Brustolin, D. y Maierna, M. y fueron empleados por este estudio (6).

\section{Tabulación}

La tabulación de la información se realizó mediante hojas de cálculo de Excel, donde se registraron los datos obtenidos (análisis de consumo de alimentos, datos bioquímicos).

\section{Análisis estadístico}

La información relacionada con salud, nutrición y alimentación se analizó de la siguiente forma: las variables cualitativas por distribuciones de frecuencia y las variables cuantitativas se describieron utilizando medidas de tendencia central (media y mediana) con desviación estándar y coeficiente de variación. El intervalo de confianza fue del 95\%. En las variables que se encontró que había solapamiento de los intervalos de confianza entre los géneros femenino y masculino se realizó la prueba t para dos muestras suponiendo varianzas desiguales, con el fin de identificar sí la diferencia entre las medias de los dos grupos es significativa o por azar. Análisis de correlación simple y múltiple se hizo para determinar el grado de relación entre las variables dependientes e independientes. En caso de no encontrar correlación se aplicó la prueba $\mathrm{Chi}^{2}$ de independencia para relacionar el consumo de carbohidratos totales, azucares y fibra. El análisis de varianza de un factor (ANOVA) se realizó para comparar los niveles de lípidos, lipoproteínas y ApoB100 con el consumo de carbohidratos totales, azúcares y fibra total, en donde el factor es el consumo y los niveles del factor son déficit, adecuado y exceso.

\section{Resultados}

De la muestra final, el $52,78 \%$ pertenece al género masculino ( 76 hombres) y el $47,22 \%$ restante al género femenino (68 mujeres), con edades promedio aproximadas de 39 y 38 años respectivamente.

\section{Perfil lipídico}

De las 144 personas tomadas como muestra, 75 fueron normolipidémicos $(52,08 \%)$ y los 69 restantes presentaron algún tipo de dislipidemia $(47,92 \%)$, destacándose en la población femenina niveles menores de triglicéridos (TG) y VLDL y niveles mayores de HDL (Tabla 1). 
Tabla 1. Niveles promedio de lípidos y lipoproteínas

\begin{tabular}{lcccc}
\hline Fracción lipídica & Masculino $(\mathbf{m g} / \mathbf{d l})$ & Femenino $(\mathbf{m g} / \mathbf{d l})$ & General $(\mathbf{m g} / \mathbf{d l})$ & Valor de referencia $(\mathbf{m g} / \mathbf{d l}) *^{*}$ \\
\hline Colesterol total & $184,94 \pm 35,8$ & $180,42 \pm 32,7$ & $182,68 \pm 34,3$ & $<200$ \\
Triglicéridos & $156,85 \pm 70,3$ & $95,33 \pm 39,2$ & $126,09 \pm 65,3$ & $<150$ \\
HDL & $41,3 \pm 9,8$ & $54,36 \pm 8,49$ & $47,83 \pm 11,3$ & $40-60$ \\
LDL & $108,5 \pm 35,6$ & $103,94 \pm 32,0$ & $106,22 \pm 33,9$ & $<130$ \\
VLDL & $31,37 \pm 14.05$ & $19,05 \pm 7,8$ & $25,21 \pm 13,0$ & $<30$ \\
\hline
\end{tabular}

$*(7)$

\section{Niveles de Apolipoproteína B100 (ApoB100)}

El valor que se tuvo como referencia fue de $30-95 \mathrm{mg} / \mathrm{dl}$, los niveles promedio del grupo estudio fueron de $84,85 \pm$ $3,7 \mathrm{mg} / \mathrm{dl}$, de las mujeres $63,50 \pm 7,6 \mathrm{y}$ de los hombres $75,82 \pm 8,5 \mathrm{mg} / \mathrm{dl}$.

\section{Consumo}

\section{Análisis cuantitativo del consumo de nutrientes}

El análisis cuantitativo del consumo de nutrientes se realizó mediante la frecuencia de consumo incluida en las encuestas realizadas en el estudio mencionado, en la tabla 2 se presenta el consumo promedio de cada nutriente, estos valores son en general más altos en el grupo masculino que en el femenino.

Para efectos del presente estudio se calculó adicionalmente la cantidad en gramos de cada tipo de carbohidratos contenidos en los alimentos consumidos habitualmente. Para esto como primera medida se clasificaron los carbohidratos, tomando como base las recomendaciones de la Food and Agriculture Organization (FAO) de las Naciones Unidas y de la World Health Organization (WHO), que en 1998, clasifican los carbohidratos según su grado de polimerización en tres grupos principales: azúcares, oligosacáridos y polisacáridos (8).

Tabla 2. Consumo promedio y desviación estándar diario de nutrientes de la muestra total y según género

\begin{tabular}{lccc}
\hline Nutriente & Masculino & Femenino & General \\
\hline Energía (Kcal) & $2743 \pm 873,97$ & $2074,07 \pm 803,88$ & $2408,54 \pm 815,50$ \\
Proteína (g) & $108,16 \pm 40,46$ & $81,81 \pm 32,28$ & $94,99 \pm 36,43$ \\
Grasa Total $(\mathrm{g})$ & $110,12 \pm 42,16$ & $86,49 \pm 34,25$ & $98,31 \pm 36,78$ \\
Colesterol (mg) & $393,12 \pm 222,2$ & $303,74 \pm 180,95$ & $348,43 \pm 200,5$ \\
Carbohidratos $(\mathrm{g})$ & $331,61 \pm 116,20$ & $255,6 \pm 121,25$ & $294,99 \pm 110,69$ \\
\hline
\end{tabular}

Tabla 3. Promedio y desviación estándar del consumo diario de carbohidratos de la muestra total y según género

\begin{tabular}{lccc}
\hline Tipo de carbohidratos & Masculino & Femenino & General \\
\hline Carbohidratos Totales(g) & $331,61 \pm 100,94$ & $255,68 \pm 74,05$ & $294,99 \pm 100,94$ \\
Almidón (g) & $59,25 \pm 25,44$ & $42,98 \pm 23,83$ & $51,80 \pm 25,44$ \\
Azúcares (g) & $118,55 \pm 41,79$ & $97,56 \pm 36,19$ & $109,78 \pm 41,79$ \\
\end{tabular}


En este caso se tuvo en cuenta la cantidad de carbohidratos totales y de ahí la cantidad según su clasificación en azúcares y polisacáridos entre estos almidón, fibra total y a su vez cada tipo de fibra. Los oligosacáridos no fueron cuantificados ya que no se encontró ninguna tabla de composición de alimentos que incluyera dentro de los nutrientes analizados algún tipo de oligosacárido. Calculada la cantidad en gramos de cada tipo de carbohidratos contenido en los alimentos consumidos habitualmente (diario) por los individuos que participaron en el estudio se promediaron y se presentan en la tabla 3.

\section{Estimación de requerimientos de energía y nutrientes}

El promedio de requerimientos de energía y nutrientes fue calculado empleando los Estimated Energy Requirementes (EERs), de esta manera se obtuvo el gasto calórico diario para cada individuo y a partir de esto se realizó la distribución porcentual recomendada de nutrientes para la población adulta sana dados por las Dietary Referentes Intakes (5), (Tablas 4 y 5).

\section{Interpretación del consumo de energía y nutrientes}

Para la evaluación e interpretación del consumo de nutrientes basados en los EERs (8), se comparó lo consumido con lo recomendado. Considerándose como "Bajo" cuando el consumo es menor del 70\%, "Adecuado" para rangos entre $70 \%$ y $100 \%$ y "Alto" para valores de consumo con porcentaje de adecuación mayor de $100 \%$.

Se observó que la distribución del consumo de energía y nutrientes es en general similar para ambos géneros, destacándose en el grupo masculino el consumo de proteínas y carbohidratos y en el femenino un consumo mayor de grasa total (Tablas 6 y 7).

Tabla 4. Promedio de recomendaciones diaria de energía y nutrientes según EER

\begin{tabular}{lccc}
\hline Nutriente & Masculino & Femenino & General \\
\hline Energía (Kcal) & 2375,99 & 1766,54 & 2071,27 \\
Proteína (g) & 89,10 & 66,25 & 77,67 \\
Grasa Total (g) & 79,20 & 58,88 & 69,04 \\
Colesterol (mg) & 200,00 & 200,00 & 200,00 \\
Carbohidratos (g) & 326,70 & 242,90 & 284,80 \\
\hline
\end{tabular}

Tabla 5. Promedio de recomendaciones diaria de carbohidratos según EER

\begin{tabular}{lccc}
\hline Tipo de carbohidratos & Masculino & Femenino & General \\
\hline Carbohidratos Totales $(\mathrm{g})$ & 170,84 & 242,98 & 280,73 \\
Azúcares $(\mathrm{g})$ & 159,42 & 110,44 & 127,60 \\
Fibra total $(\mathrm{g})$ & 204,14 & 23,00 & 27,71 \\
\hline
\end{tabular}

Tabla 6. Interpretación de consumo de nutrientes según ERRs en el grupo masculino

\begin{tabular}{|c|c|c|c|c|c|c|c|c|c|c|}
\hline \multirow[t]{2}{*}{ Interpretación } & \multicolumn{2}{|c|}{ Energía } & \multicolumn{2}{|c|}{ Proteína } & \multicolumn{2}{|c|}{ Grasa total } & \multicolumn{2}{|c|}{ CHO } & \multicolumn{2}{|c|}{ Colesterol } \\
\hline & \# & $\%$ & \# & $\%$ & \# & $\%$ & \# & $\%$ & \# & $\%$ \\
\hline Alto & 50 & 65,7 & 47 & 61,8 & 56 & 73,7 & 41 & 63 & 63 & 82 \\
\hline Adecuado & 21 & 27,6 & 22 & 28,9 & 17 & 22,3 & 23 & 13 & 13 & 17 \\
\hline Bajo & 5 & 6,5 & 7 & 9,2 & 3 & 3,9 & 12 & 0 & 0 & 0 \\
\hline Total & 76 & 100 & 76 & 100 & 76 & 100 & 76 & 76 & 76 & 100 \\
\hline
\end{tabular}


Tabla 7. Interpretación de consumo de energía y nutrientes según ERR en el grupo femenino

\begin{tabular}{lcccccccccc}
\hline Interpretación & \multicolumn{2}{c}{ Energía } & \multicolumn{2}{c}{ Proteína } & \multicolumn{2}{c}{ Grasa total } & \multicolumn{2}{c}{ CHO } & \multicolumn{2}{c}{ Colesterol } \\
\hline & $\#$ & $\%$ & $\#$ & $\%$ & $\#$ & $\%$ & $\#$ & $\%$ & $\#$ & $\%$ \\
\hline Alto & 47 & 69,7 & 46 & 27,6 & 59 & 86,7 & 35 & 51,5 & 52 & 76,4 \\
Adecuado & 18 & 26,5 & 19 & 27,9 & 8 & 11,7 & 21 & 30,9 & 16 & 23,5 \\
Bajo & 3 & 4,4 & 3 & 4,41 & 1 & 1,47 & 12 & 17,7 & 0 & 0 \\
Total & 68 & 100 & 68 & 100 & 68 & 100 & 68 & 100 & 68 & 100 \\
\hline
\end{tabular}

\section{Interpretación del consumo de carbohidratos}

En la muestra total se encontró que el porcentaje más alto de la población, el $52,78 \%$, tiene alto consumo de carbohidratos totales, mientras que en azúcares y fibra total el mayor porcentaje de la población presentan consumos bajos con un $44,44 \%$ y $77,78 \%$ respectivamente (Tabla 8 ).

Analizando los géneros por separado, se encontró que los hombres tienen alto consumo de carbohidratos totales, el $48,68 \%$ tiene bajo consumo de azúcares y la mayoría tiene adecuado consumo de fibra total, en el grupo femenino, también se observa alto consumo de carbohidratos, el consumo de azúcares y de fibra total en la mayoría es bajo.

\section{Relación de variables}

\section{Prueba t para diferencia de medias}

Esta prueba se realizó en aquellos casos en que se encontró solapamiento entre los intervalos de confianza en los géneros, esta situación se presento en los siguientes casos:

- Consumo de azúcares: realizada la prueba t para la diferencia entre el consumo promedio de azúcares en los géneros masculino y femenino, se encontró que hay diferencias en el consumo de azúcares entre estos, y que este consumo es significativamente mayor en hombre que en mujeres.

- Niveles de triglicéridos, VLDL y HDL: aquí se encontró que estos son significativamente diferentes entre el género masculino y femenino, presentándose mayores niveles en el masculino de triglicéridos y VLDL, mientras que en el femenino son mayores los niveles de HDL.

\section{Análisis de correlación}

Se realizó correlación simple de Pearson (r), cuyos valores de correlación varían entre +1 a -1 , pasando por el cero, el cual corresponde a ausencia de correlación. Para determinar la intensidad de la relación entre las variables cuantitativas de interés, los cruces de variables se realizaron de diversas formas, en primer lugar se relacionó el consumo en gramos de cada tipo de carbohidratos, con los niveles de lípidos, lipoproteínas y ApoB100, en donde no se encontró correlación para ninguno de los casos, es decir, la cantidad en gramos de cada tipo de carbohidratos consumidos no tiene relación con los niveles de lípidos plasmáticos (Tabla 9).

Tabla 8. Interpretación del consumo de carbohidratos en la muestra estudio

\begin{tabular}{|c|c|c|c|c|c|c|}
\hline \multirow[t]{2}{*}{$\begin{array}{l}\text { Interpretación } \\
\text { del consumo }\end{array}$} & \multicolumn{2}{|c|}{$\begin{array}{c}\text { CHOs total } \\
\text { (g) }\end{array}$} & \multicolumn{2}{|c|}{$\begin{array}{c}\text { Azúcares } \\
\text { (g) }\end{array}$} & \multicolumn{2}{|c|}{$\begin{array}{c}\text { Fibra total } \\
\text { (g) }\end{array}$} \\
\hline & \# & $\%$ & \# & $\%$ & $\#$ & $\%$ \\
\hline Bajo & 25 & 17,36 & 64 & 44,44 & 112 & 77,78 \\
\hline Adecuado & 43 & 29,86 & 33 & 22,92 & 21 & 14,58 \\
\hline Alto & 76 & 52,78 & 47 & 32,64 & 11 & 7,64 \\
\hline Total & 144 & 100 & 144 & 100 & 144 & 100 \\
\hline
\end{tabular}


Por último se relacionaron los niveles plasmáticos de la ApoB100 con cada una de las fracciones lipídicas, encontrándose, correlaciones moderadas entre la ApoB100 y el colesterol total $(\mathrm{r}=0,76)$, e igualmente la ApoB100 y las LDL ( $\mathrm{r}=0,70)$ (Tabla 10).

\section{Prueba Chi ${ }^{2}$}

Se utilizo la prueba $\mathrm{Chi}^{2}$ para determinar la asociación entre los niveles plasmáticos de lípidos, lipoproteínas y apolipoproteína B100 y el consumo de carbohidratos totales, azucares y fibra total, no encontrándose asociación en ninguna de las variables ( $p>0,05)$.

\section{Análisis de varianza de un factor (ANOVA)}

Luego de determinar que los datos tenían una distribución normal se procedió a realizar un ANOVA para comparar los niveles de lípidos, lipoproteínas y apolipoproteína B100 según el consumo de carbohidratos totales, azúcares y fibra total.

Luego de realizar esta prueba se encontró, no hay diferencias significativas entre los niveles promedio de lípidos, lipoproteínas y Apo B100 y consumo bajo, adecuado o alto de los diferentes tipos de carbohidratos $(\mathrm{p}>0,05)$.

\section{Discusión}

La alimentación y el perfil lipídico son factores que juegan un papel muy importante en el desarrollo de enfermedad cardiovascular, estos dos son las variables principales analizadas en este estudio.

Analizando el perfil lipídico como variable dependiente en este estudio, según genero se encontró que las concentraciones promedio de colesterol total, LDL, triglicéridos y VLDL fueron mayores en el grupo de los hombres que en el de las mujeres, mientras que los de HDL fueron mayores en las mujeres, esto refleja que en este grupo estudio que presenta edades promedio de 39 en hombres y 38 en mujeres, las mujeres presentan niveles plasmáticos de su perfil lipídico mejor que los hombres, especialmente antes de llegar a la menopausia, esta tendencia también es descrita por la Sociedad Española de Aterosclerosis, que encontró que las

Tabla 9. Coeficientes de correlación de Pearson (r) entre el consumo de Carbohidratos totales, Almidón, Azúcares, Fibra total. Fibra soluble y Fibra insoluble con los niveles plasmáticos de lípidos, lipoproteínas y ApoB100

\begin{tabular}{lcccccc}
\hline Perfil lipídico & CHOs totales & Almidón & Azúcares & Fibra total & Fibra soluble & Fibra insoluble \\
\hline ApoB100 & 0,02 & 0,04 & 0,05 & 0,05 &, 08 & 0,08 \\
Colesterol Total & 0,18 & 0,02 & 0,005 & 0,19 & 0,06 & 0,04 \\
Triglicéridos & 0,25 & 0,10 & 0,22 & 0,01 & 0,01 & 0,01 \\
HDL & 0,31 & 0,14 & 0,27 & 0,09 & 0,11 & 0,10 \\
LDL & 0,05 & 0,05 & 0,015 & 0,03 & 0,07 & 0,05 \\
VLDL & 0,25 & 0,10 & 0,22 & 0,01 & 0,14 & 0,01 \\
\hline
\end{tabular}

Tabla 10. Coeficientes de correlación de Pearson (r) entre los niveles de ApoB100 y los niveles plasmáticos de lípidos, lipoproteínas.

\begin{tabular}{lccc}
\hline Perfil lipídico & \multicolumn{3}{c}{ Coeficiente de correlacion } \\
\hline & Masculino & Femenino & Población general \\
\hline Colesterol total & 0,74 & 0,63 & 0,76 \\
Trigliceridos & 0,12 & 0,08 & 0,12 \\
HDL & 0,16 & 0,09 & 0,11 \\
LDL & 0,66 & 0,64 & 0,70 \\
VLDL & 0,12 & 0,09 & 0,12 \\
\hline
\end{tabular}


mujeres adultas tienen niveles mas altos de HDL mientras que los hombres presentan niveles mayores de colesterol total y LDL, lo cual puede llevar a tener mayor riesgo de desarrollar Enfermedades Cardiovasculares (9).

Estas diferencias entre géneros también se evidencian en el consumo, al cuantificar, evaluar y comparar el consumo de los diferentes tipos de carbohidratos entre los dos géneros se encontraron diferencias, pero, solo en el caso de los azúcares se encontraron diferencias significativas con un $\mathrm{p}<0,005$ entre géneros, presentando mayor consumo los hombres con respecto a las mujeres, hecho que podría influir en sus niveles también mayores de triglicéridos plasmáticos, tendiendo en cuenta que lo encontrado en la literatura reporta que las dietas pobres en grasa y con altos contenidos de carbohidratos elevan los niveles de triglicéridos por un aumento de la síntesis hepática de triglicéridos. Este proceso no se acompaña de un aumento en la síntesis de esteres de colesterol ni de ApoB100, por lo que los niveles plasmáticos de VLDL no varia. Además las dietas ricas en carbohidratos reducen la actividad de la lipoprotein lipasa induciendo hipertrigliceridemia. En cualquier caso la hipertrigliceridemia inducida por el exceso de carbohidratos es de duración limitada en el tiempo, volviendo los niveles de triglicéridos a valores normales en un periodo de dos a cuatro semanas cuando el individuo no presenta ninguna otra patología metabólica asociada con es la resistencia a la insulina (10).

Contrario a lo reportado en la investigación de Schaefer (11), donde se encontró que los niveles elevados de VLDL, LDL y ApoB100, estaban influenciados en gran parte por la alimentación, especialmente por el tipo y cantidad de carbohidratos y grasas, en este estudio se encontró esta relación entre la LDL y la ApoB100, mediante tres pruebas estadísticas, tales como, el análisis de correlación, la prueba $\mathrm{Chi}^{2}$ y el ANOVA se pudo evidenciar que no hay relación entre el tipo y la cantidad de carbohidratos consumidos con los niveles plasmáticos de VLDL, LDL y ApoB100, tampoco con las demás fracciones lipídicas.

Otras investigaciones sugieren que el aumento de ApoB100 también puede darse según el tipo y la cantidad de carbohidratos en la dieta (12), luego de cuantificar la cantidad de cada tipo de carbohidratos contenido en los alimentos de consumo habitual en los individuos participantes en el estudio y compararlo con los niveles de ApoB100, no se encontró ninguna relación significativa, independientemente del tipo de carbohidratos, sean carbohidratos totales, azúcares, almidón, fibra total, soluble e insoluble, ninguno tiene relación con los niveles plasmáticos de ApoB100, hecho contrario a lo que se ha encontrado en otra investigación que aunque no es en humanos, compara el tipo de carbohidratos y los niveles plasmáticos de colesterol en monos, donde se muestra evidencia que en una dieta que contiene niveles altos de grasa saturada (40\% del valor calórico total) y $1 \mathrm{mg} / \mathrm{Kcal}$ de colesterol, el almidón es claramente más hipercolesterolémico que la sacarosa (12).

La ApoB100 es la principal apolipoproteína de las VLDL, IDL, y especialmente de las LDL, ya que hacen parte del $95 \%$ del total de sus proteínas (13). Esta afirmación se pudo observar en el estudio, al relacionar los niveles plasmáticos de apo B100 con cada una de las fracciones lipídicas, encontrándose, como era de esperarse correlaciones significativas entre la ApoB100 y el colesterol total, e igual entre la ApoB100 y las LDL.

\section{Conclusión}

No se encontró relación significativa entre el tipo y la cantidad de carbohidratos consumidos por los individuos estudiados con los niveles plasmáticos de lípidos, lipoproteínas y Apo B100.

\section{Financiación}

Este trabajo hace parte de un proyecto financiado por la Pontificia Universidad Javeriana, código 1589.

\section{Conflicto de intereses}

Los autores declaran que no existen conflictos de intereses con los resultados de este trabajo.

\section{Referencias}

1. Olofsson $\mathrm{O}$, Borèn $\mathrm{J}$. Apolipoprotein B: a clinically important apolipoprotein which assembles atherogenic lipoprotein and promotes the development of atherosclerosis. Journal of Internal Medicine. 2005; 258: 395-410.

2. Dolan LC, Potter SM, Burdock GA. Evidence-Base Review on the effect of normal dietary consumption of fructose on development of hyperlipidemia and obesity in healthy, normal weight individuals. Critical Reviews in Food Science and Nutrition. 2010; 50:53-84. 
3. Ojeda M, Torres A, Guerra M. Influencia de la dieta sobre los niveles plasmáticos de lípidos en sujetos adultos sanos con diferentes genotipos del gen de la FABP2. Tesis de Maestría. Facultad de Ciencias. Universidad Javeriana, Colombia, 2005.

4. Centro de Atención Nutricional. Tabla de Composición de los Alimentos. Medellín, Colombia. 2003.

5. National Academy of Sciences. Institute of Medicine. Dietary Reference Intakes for Energy, Carbohydrate, Fiber, Fat, Fatty Acids, Cholesterol, Protein, and Amino Acids (Macronutrients). Food and Nutrition Board. Washington D.C. USA. 2005.

6. Brustolin D, Maierna M, Aguzzi F, Zoppi F, Tarenghi $\mathrm{G}$, Immunoturbidimetric method for routine determinations of apolipoproteins A-I and B. Clinical Chemistry. 1991; 37: 742-747.

7. Cleeman J. Executive Summary of the This Report of the National Education Program (NCEP) Expert Panel on Detection, Evaluation, and Treatment of High Blood Cholesterol in Adults (Adult Treatment Panel III). Journal of the American Dietetic Association. 2001; 102: 2486 - 2496.

8. Food and Agriculture Organization of de United Nations and World Health Organization. Report of a joint FAO/WHO Expert consultation: Carbohydrates in Human Nutrition. Roma, Italia. 1998.

9. Sociedad Española de Arteriosclerosis, Sociedad Española de Medicina Interna y Liga de Lucha contra la Hipertensión Arterial. Recomendaciones para la prevención primaria de la enfermedad cardiovascular. Ciencia e Investigación en Arteriosclerosis. 1994; 6, 62-102.

10. Gibney M. Metabolismo y Nutrición. Editorial Acribia. Zaragoza, España. 2003, 252 - 265 p.

11. Schaefer E. Lipoproteins, nutrition and heart disease. The American Journal of Clinical Nutrition. 2002; 75: 191-212.

12. Srinivasan S. Serum lipoprotein lipid and apoprotein (B and A-1) responses to saturated fat- rich diet in cynomolgus monkeys: effects of carbohydrate type and cholesterol. The American Journal of Clinical Nutrition. 1984; 40:485 - 495.

13. Hooper A, Bockxmer VF, Burnett J. Monogenic hypocholesterolemic lipid disorders and apolipoprotein B metabolism. Clinical Reviews in Clinical Laboratory Science. 2005; 42: 515 - 535. 